\title{
THE RARE 23.1-GHZ METHANOL MASERS IN NGC 7538 IRS 1
}

\author{
Roberto Galván-Madrid ${ }^{1,2,3}$, Gabriela Montes $^{2,4}$, Edgar A. Ramírez ${ }^{2,5}$, \\ Stan Kurtz ${ }^{2}$, Esteban Araya ${ }^{6}$, and Peter Hofner ${ }^{7,8,9}$ \\ rgalvan@cfa.harvard.edu
}

\begin{abstract}
We present high angular resolution $\left(\theta_{\text {syn }} \lesssim 0\right.$ ". 2$)$ observations of the $23.1-\mathrm{GHz}$ methanol $\left(\mathrm{CH}_{3} \mathrm{OH}\right)$ transition toward the massive star forming region NGC 7538 IRS 1. The two velocity components previously reported by Wilson et al. are resolved into distinct spatial features with brightness temperatures $\left(T_{B}\right)$ greater than $10^{4} \mathrm{~K}$, proving their maser nature. Thus, NGC 7538 IRS 1 is the third region confirmed to show methanol maser emission at this frequency. The brighter 23.1-GHz spot coincides in position with a rare formaldehyde $\left(\mathrm{H}_{2} \mathrm{CO}\right)$ maser, and marginally with a $22.2-\mathrm{GHz}$ water $\left(\mathrm{H}_{2} \mathrm{O}\right)$ maser, for which we report archival observations. The weaker $\mathrm{CH}_{3} \mathrm{OH}$ spot coincides with an $\mathrm{H}_{2} \mathrm{O}$ maser. The ratio of $T_{B}$ for the 23.1-GHz masers to that of the well-known $12.2-\mathrm{GHz} \mathrm{CH}_{3} \mathrm{OH}$ masers in this region roughly agrees with model predictions. However, the 23.1-GHz spots are offset in position from the $\mathrm{CH}_{3} \mathrm{OH}$ masers at other frequencies. This is difficult to interpret in terms of models that assume that all the masers arise from the same clumps, but it may result from turbulent conditions within the gas or rapid variations in the background radiation field.
\end{abstract}

Subject headings: H II regions — ISM: individual (NGC 7538) — ISM: molecules — masers - radio lines - stars: formation

\footnotetext{
${ }^{1}$ Harvard-Smithsonian Center for Astrophysics, 60 Garden Street, Cambridge MA 02138, USA

${ }^{2}$ Centro de Radioastronomía y Astrofísica, Universidad Nacional Autónoma de México, Morelia 58090, México

${ }^{3}$ Academia Sinica Institute of Astronomy and Astrophysics, P.O. Box 23-141, Taipei 106, Taiwan

${ }^{4}$ Instituto de Astrofísica de Andalucía, CSIC, Camino Bajo de Huétor 50, E-18008 Granada, España

${ }^{5}$ Department of Physics and Astronomy, University of Sheffield, Sheffield S3 7RH, England

${ }^{6}$ Physics Department, Western Illinois University, 1 University Circle, Macomb IL 61455, USA

${ }^{7}$ Physics Department, New Mexico Tech, 801 Leroy Place, Socorro NM 87801, USA

${ }^{8}$ National Radio Astronomy Observatory, P.O. Box 0, Socorro NM 87801, USA

${ }^{9}$ Max-Planck-Institut für Radioastronomie, Auf dem Hügel 69,53121, Bonn, Germany
} 


\section{Introduction}

NGC 7538 is a Galactic star forming region located at a distance of $2.65_{-0.11}^{+0.12} \mathrm{kpc}$ (Moscadelli et al. 2009). Multiple infrared sources were discovered in the vicinity by Wynn-Williams et al. (1974). The brightest of these, IRS 1, was recently resolved in the near-IR and mid-IR by Kraus et al. (2006) and De Buizer \& Minier (2005), respectively. The centimeter free-free emission has a bipolar structure (Campbell 1984; Sandell et al. 2009; Zhu et al. 2010) and shows variability (Franco-Hernández \& Rodríguez 2004). Also, the recombination lines from the ionized gas are unusually broad (Gaume et al. 1995; Sewiło et al. 2004; Keto et al. 2008).

NGC 7538 IRS 1 is an exceptionally rich maser source. Maser emission has been detected in hydroxyl $(\mathrm{OH})$, water $\left(\mathrm{H}_{2} \mathrm{O}\right)$, ammonia $\left(\mathrm{NH}_{3}\right)$, methanol $\left(\mathrm{CH}_{3} \mathrm{OH}\right)$, and formaldehyde $\left(\mathrm{H}_{2} \mathrm{CO}\right)$ (e.g., Gaume et al. 1991, hereafter G91; Hutawarakorn \& Cohen 2003; Hoffman et al. 2003, hereafter H03; Kurtz et al. 2004). $\mathrm{CH}_{3} \mathrm{OH}$ emission at $23.1 \mathrm{GHz}$, from the $9_{2}-10_{1} A^{+}$transition, was reported for NGC 7538 by Wilson et al. (1984) (hereafter W84). They speculated that the emission was maser in nature, based on observations taken with the Effelsberg radio telescope $\left(\theta_{\text {beam }} \approx 43^{\prime \prime}\right)$. The well-known $6.7-\mathrm{GHz}\left(5_{1}-6_{0} A^{+}\right)$and $12.2-\mathrm{GHz}\left(2_{0}-3_{-1} E\right)$ class II $\mathrm{CH}_{3} \mathrm{OH}$ masers have also been detected toward this source (Minier et al. 2000, hereafter M00; Minier et al. 2002; Moscadelli et al. 2009, hereafter M09). Evidence for a circumstellar disk in IRS 1, based on a velocity gradient within a linear structure of $6.7-\mathrm{GHz}$ and $12.2-\mathrm{GHz}$ methanol maser spots, was presented by Pestalozzi et al. (2004, 2009) (see however De Buizer \& Minier 2005). The warm molecular gas surrounding the hypercompact H II region (IRS 1) also has dynamics indicative of rotation (Klaassen et al. 2009).

The 23.1- $\mathrm{GHz}$ class II $\mathrm{CH}_{3} \mathrm{OH}$ maser is quite rare. Until now, only two regions harboring these masers have been confirmed: W3(OH) and NGC $6334 \mathrm{~F}$ (W84; Menten et al. 1985; Menten et al. 1988; Menten \& Batrla 1989). Cragg et al. (2004) observed 50 southern star-forming regions and detected 23.1-GHz maser emission in only one - the previously known NGC $6334 \mathrm{~F}$. The 4.8-GHz $\left(1_{10}-1_{11}\right) \mathrm{H}_{2} \mathrm{CO}$ maser in NGC 7538 IRS 1 is also quite rare; at present, only seven of these masers have been found in the Galaxy (Araya et al. 2008, and references therein). And $\mathrm{NH}_{3}$, although ubiquitous in massive star forming regions, is not a common maser. Various transitions and isotopes are known to present maser emission (e.g., Schilke et al. 1991; Hofner et al. 1994; Galván-Madrid et al. 2009), but to date, the metastable ${ }^{15} \mathrm{NH}_{3}(3,3)$ maser has only been found in NGC 7538 IRS 1 (G91; Mauersberger et al. 1986; Johnston et al. 1989; Wyrowski \& Walmsley 1996).

Because the original 23.1- $\mathrm{GHz} \mathrm{CH}_{3} \mathrm{OH}$ detection by W84 was never pursued at higher angular resolution, and because of the presence in IRS 1 of not one but possibly three rare maser species, we observed NGC 7538 IRS 1 with the goals of confirming the maser nature of the $23.1-\mathrm{GHz} \mathrm{CH}_{3} \mathrm{OH}$

emission, and accurately locating this emission with respect to the other masers. In $\S 2$ we describe the observations and the data reduction procedure. We present the results in $\S 3$ and provide a discussion in $\S 4$. 


\section{Observations}

We observed the $\mathrm{CH}_{3} \mathrm{OH} 9_{2}-10_{1} A^{+}$transition at $1.3 \mathrm{~cm}\left(\nu_{0}=23.121024 \mathrm{GHz}\right.$, Mehrotra et al. 1985) toward NGC 7538 IRS 1 with the VLA of NRAO 1 The observations were made on 2006 May 29 and May 30 for a total on-source time of $\sim 1$ hour (program AG722). The array was in the BnA configuration and the pointing center was $\alpha(\mathrm{J} 2000)=23^{\mathrm{h}} 13^{\mathrm{m}} 46^{\mathrm{s}} \cdot 00, \delta(\mathrm{J} 2000)=61^{\circ} 28^{\prime} 11^{\prime \prime} \cdot 0$. We used the $1 \mathrm{~A}$ correlator mode, measuring right circular polarization without Hanning smoothing. A $3.125 \mathrm{MHz}$ bandwidth with 255 channels of $12.207 \mathrm{kHz}$ each, provided a velocity coverage of $40 \mathrm{~km}$ $\mathrm{s}^{-1}$ and a channel width of $0.16 \mathrm{~km} \mathrm{~s}^{-1}$.

The data were reduced following standard spectral line procedures with the AIPS software of NRAO. The flux, phase, and bandpass calibrators were J0137+331 $\left(S_{\nu}=1.08 \mathrm{Jy}\right.$, calculated using the 1999.2 VLA values), J2322+509 $\left(S_{\nu}=0.93 \pm 0.02 \mathrm{Jy}\right.$, bootstrapped flux density), and J0319+415 $\left(S_{\nu}=11.4 \pm 0.2 \mathrm{Jy}\right.$, bootstrapped flux density), respectively. The data received one iteration of phase-only self-calibration. A continuum level of about $300 \mathrm{mJy}$ was subtracted in the $(u, v)$ plane and bandpass calibration was applied. The final image cube was made with the ROBUST parameter of AIPS task IMAGR set to 0 , resulting in a synthesized beam of FWHM = 0 " $23 \times 0$ ". 12 at position angle $+70^{\circ}$. The per-channel noise level of the CLEANed image cube was $7 \mathrm{mJy}^{\text {beam }}{ }^{-1}$.

The highest angular resolution observations of $\mathrm{H}_{2} \mathrm{O}$ masers in NGC 7538 reported in the literature are the 5-6" resolution data of Kameya et al. (1990). However, archival A-configuration VLA data, taken on 1999 July 30 under program AS667 (see Sarma et al. 2002) are available. The data were taken in B1950 coordinates, and were precessed to J2000 before processing. The precessed pointing center was $\alpha=23^{\mathrm{h}} 13^{\mathrm{m}} 45.333, \delta=61^{\circ} 28^{\prime} 10^{\prime \prime} .59$. The observations were made in the $4 \mathrm{IF}$ Hanning-smoothed mode, observing the water maser line at $22.235 \mathrm{GHz}$, centered at $\mathrm{V}_{L S R}=-58$ $\mathrm{km} \mathrm{s}^{-1}$. A $0.781 \mathrm{MHz}$ bandwidth with 63 channels was employed, providing a velocity coverage of $10.5 \mathrm{~km} \mathrm{~s}^{-1}$ with $0.17 \mathrm{~km} \mathrm{~s}^{-1}$ channels.

The data were reduced using standard high-frequency calibration procedures. The flux and phase calibrators were B0134+329 $\left(S_{\nu}=1.13 \mathrm{Jy}\right.$, calculated from the $1992.2 \mathrm{VLA}$ parameters $)$ and B2320+506 $\left(S_{\nu}=1.33 \pm 0.07 \mathrm{Jy}\right.$, bootstrapped flux $)$. No bandpass calibration was applied. The data were self-calibrated in phase and three fields were imaged with a synthesized beam of 99.6 $\times 77.1$ milli-arcsec at a position angle of $+15^{\circ}$. Maser-free channels had a typical rms noise of 20

mJy beam ${ }^{-1}$. One field was 1'.3 from the pointing center; a primary beam correction was applied to this image.

Positional uncertainty arises from the intrinsic uncertainty in the calibrator position $(<0$ "'002 for B2320+506), phase stability and distance from the phase tracking center, precession of coordinates, and the statistical uncertainty of the Gaussian fit. The latter quantity depends on the

\footnotetext{
${ }^{1}$ The National Radio Astronomy Observatory is a facility of the National Science Foundation operated under cooperative agreement by Associated Universities, Inc.
} 
signal-to-noise ratio and varies from one maser to another. Taking the sources of uncertainty into account, we report a worst-case absolute positional error for the water masers of 0 !' 05 .

\section{Results}

\subsection{Methanol Masers: Source Sizes and Positions}

We detect emission at two sky positions, and denote them by $N$ (for North) and $S$ (for South). The two features are nominally resolved at our angular resolution. The emission in the peak channel of each component was well-fit by a 2 -D Gaussian. The $S$ component has a deconvolved size of $00^{\prime \prime} 11 \times 0 . \prime 07\left( \pm 0{ }^{\prime \prime} 05\right)$ at position angle $+73^{\circ} \pm 40^{\circ}$. The $N$ component has a major axis of $0 .^{\prime \prime} 09$ (at position angle $+49^{\circ} \pm 10^{\circ}$ ); it is unresolved in the orthogonal direction.

The spatial positions of the two spectral components are separated by about a beamwidth (see Figure 1). The 2-D Gaussian fits yield J2000 positions of $\alpha_{N}=23^{\mathrm{h}} 13^{\mathrm{m}} 45.358, \delta_{N}=61^{\circ} 28^{\prime} 10^{\prime \prime} .45$; and $\alpha_{S}=23^{\mathrm{h}} 13^{\mathrm{m}} 45.365, \delta_{S}=61^{\circ} 28^{\prime} 10^{\prime \prime} .28$ for the $N$ and $S$ components, respectively. The absolute position of the phase calibrator $(\mathrm{J} 2322+509)$ is reported with an accuracy of $0.002^{\prime \prime}$ in the VLA database. The phase noise in this quasar after calibration was $\lesssim 10^{\circ}$, and no change in the position of the maser spots was introduced by the self-calibration. We estimate the absolute positional uncertainty to be not greater than 0. " 05 .

\subsection{Methanol Masers: Spectral Line Parameters}

We fit the spectrum of each emission spot with a single Gaussian profile (see Fig. 2), to obtain the line-center velocities, linewidths, and peak intensities listed in Table 1 . These results are roughly consistent with those of W84. The flux densities of the spectral features are lower by $26 \%$ and $54 \%$ (for components $\mathrm{N}$ and $\mathrm{S}$ ) than in the Effelsberg observations (W84). Also, the linewidths at half power in our data are $32 \%$ smaller $(\mathrm{N})$ and $52 \%$ larger $(\mathrm{S})$ than in W84. The centroid velocities match within $0.3 \mathrm{~km} \mathrm{~s}^{-1}$ with those reported by W84. The discrepancies could arise for a number of reasons, such as time variability in the masers, different Gaussian fitting procedures, and our higher spatial/spectral resolution and signal-to-noise ratios.

\subsection{Water Masers}

Twenty-one water masers were detected in three distinct clusters. We refer to these clusters as M (main; coincident with IRS 1), E (east, about $6^{\prime \prime}$ east of IRS 1 ), and S (south, about 80" south of IRS 1 and corresponding to NGC 7538 IRS 11). The position, peak flux, velocity, and velocity range of each maser are reported in Table 2. Within each cluster the masers are numbered by increasing right ascension. The positions of some of the masers corresponding to IRS 1 are shown 
in Figure 3.

Water masers frequently occur over a wide velocity range and indeed, maser emission was found at both extremes of the $0.781 \mathrm{MHz}$ passband. Kameya et al. (1990) reported a velocity range of -45.4 to -83.4 for water masers around IRS $1-3$; it is highly probable that additional masers are present at velocities outside of the -52.8 to $-62.2 \mathrm{~km} \mathrm{~s}^{-1}$ range observed by program AS667.

\section{Discussion}

\subsection{Nature of the emission}

W84 did not have sufficient angular resolution to place a useful limit on the source brightness temperature. Nevertheless, they argued that the emission was of maser origin, based on absorption in the $\mathrm{CH}_{3} \mathrm{OH} 10_{1}-9_{2} A^{-}$line. This absorption implied that the excitation temperature of the $\mathrm{CH}_{3} \mathrm{OH} 9_{2}-10_{1} A^{+}$emission line was greater than the background temperature of at least $1000 \mathrm{~K}$. With our 250× higher angular resolution we calculate brightness temperatures of $T_{N}>1.3 \times 10^{5}$ $\mathrm{K}$ and $T_{S} \sim 4 \times 10^{4} \mathrm{~K}$ for components $N$ and $S$, respectively. Such high brightness temperatures cannot reflect the kinetic temperature of the molecular gas, thus we prove the maser nature of the emission. The relatively broad linewidths may indicate that the masers are saturated; alternatively, it could indicate the presence of unresolved maser components.

Three objects are now confirmed to harbor 23.1-GHz $\mathrm{CH}_{3} \mathrm{OH}$ maser emission: NGC 7538 IRS

1 (this paper), W3(OH) (Menten et al. 1985, 1988), and NGC 6334F (Menten \& Batrla 1989).

\subsection{Comparison with other masers}

Figure 3 shows an overlay of the free-free continuum with the locations of the masers we discuss here. The 6.7-GHz and $12.2-\mathrm{GHz} \mathrm{CH}_{3} \mathrm{OH}$ masers reported by $\mathrm{M} 00$ (right panel) and the 12.2- $\mathrm{GHz} \mathrm{CH}_{3} \mathrm{OH}$ masers reported by $\mathrm{M} 09$ (left panel) are plotted in separate panels for clarity. The positions of M00 were shifted by 18 mas to the west and south to account for the proper motions reported by $\mathrm{M} 09$ for $\mathrm{CH}_{3} \mathrm{OH}$ masers. Although not shown in Figure 3, OH masers reported by Hutawarakorn \& Cohen (2003) are located slightly south of the IRS 1 core, coincident with the more diffuse continuum emission seen at the bottom of Figure 3. The most striking feature is the general clustering of the many different maser species around IRS 1 . The bright core of continuum emission from IRS 1 has linear dimensions of order 1000 AU. Scattered across this area are the various masers of class II $\mathrm{CH}_{3} \mathrm{OH}, \mathrm{H}_{2} \mathrm{O}, \mathrm{NH}_{3}$, and $\mathrm{H}_{2} \mathrm{CO}$. There are three spatial coincidences at the present angular resolutions. One is the $23.1-\mathrm{GHz}$ component $S$, which coincides with $\mathrm{H}_{2} \mathrm{O}$ maser M8. Another is the 23.1-GHz component $N$, which coincides with component I of the 4.8$\mathrm{GHz}$ formaldehyde maser (which is itself resolved into two components: Ia and Ib, separated by 
$\sim 60 \mathrm{AU}$; H03) and is also marginally associated with the $\mathrm{H}_{2} \mathrm{O}$ maser M7. The third association is $\mathrm{H}_{2} \mathrm{O}$ maser $\mathrm{M} 6$ with component II of the $\mathrm{H}_{2} \mathrm{CO}$ masers. Table 3 lists the positions, offsets, and absolute positional uncertainty of the coincident masers. The 6.7 or $12.2-\mathrm{GHz} \mathrm{CH}_{3} \mathrm{OH}$ spot closest to a 23.1-GHz maser is component 1 of Moscadelli et al. (2009), with a separation of 65 mas (or $1.3 \sigma$ ) from our component $N$. As we are confident with our estimates of the astrometric precision, we still rule out an association between these features.

The velocities of the 23.1-GHz $\mathrm{CH}_{3} \mathrm{OH}$ masers $\left(V_{N} \approx-56.1 \mathrm{~km} \mathrm{~s}^{-1}, V_{S} \approx-59.1 \mathrm{~km} \mathrm{~s}^{-1}\right)$ are similar to those of the other masers in the vicinity of IRS 1 . The $6.7-\mathrm{GHz}^{\mathrm{CH}} \mathrm{H}_{3} \mathrm{OH}$ masers have LSR velocities in the range $V_{6.7}=[-61.6,-55.0] \mathrm{km} \mathrm{s}^{-1}$, and the cross-power spectrum of those masers for the entire field shows two velocity components centered at $\approx-56 \mathrm{~km} \mathrm{~s}^{-1}$ and

$\approx-58 \mathrm{~km} \mathrm{~s}^{-1}$ (M00). M00 also report several 12.2-GHz $\mathrm{CH}_{3} \mathrm{OH}$ masers coincident with 6.7-GHz masers; the velocities of the former are $\approx-58 \mathrm{~km} \mathrm{~s}^{-1}$. M09 reports more $\mathrm{CH}_{3} \mathrm{OH}$ masers at $12.2 \mathrm{GHz}$ than does $\mathrm{M} 00$; all of the additional masers are in the velocity interval $[-61.9,-55.8]$ $\mathrm{km} \mathrm{s}^{-1}$. The $4.8-\mathrm{GHz} \mathrm{H}_{2} \mathrm{CO}$ masers also have two velocity components. The components Ia and Ib have almost the same LSR velocity $\left(V_{I a} \approx-57.9 \mathrm{~km} \mathrm{~s}^{-1}, V_{I b} \approx-57.8 \mathrm{~km} \mathrm{~s}^{-1}\right)$, while component II is at $V_{I I} \approx-60.2 \mathrm{~km} \mathrm{~s}^{-1}(\mathrm{H} 03)$. The ${ }^{15} \mathrm{NH}_{3}(3,3)$ masers have velocities in the range $V_{15} \mathrm{NH}_{3}=[-52.8,-61.7] \mathrm{km} \mathrm{s}^{-1}$ (G91). The $\mathrm{H}_{2} \mathrm{O}$ masers reported here have a velocity range of $V_{\mathrm{H}_{2} \mathrm{O}}=[-53,-63] \mathrm{km} \mathrm{s}^{-1}$ (see Table 1). The typical errors in the determination of the velocity features are $\sim 0.1 \mathrm{~km} \mathrm{~s}^{-1}$ or better.

For the positional associations the corresponding velocities are very similar. $\mathrm{CH}_{3} \mathrm{OH}$ maser $S$ is centered at $-59.11 \mathrm{~km} \mathrm{~s}^{-1}$, and $\mathrm{H}_{2} \mathrm{O}$ maser $\mathrm{M} 8$ spans the range $[-60.8,-59.2] \mathrm{km} \mathrm{s}^{-1} \cdot \mathrm{CH}_{3} \mathrm{OH}$ maser $N$ is centered at $-56.09 \mathrm{~km} \mathrm{~s}^{-1}$ while $\mathrm{H}_{2} \mathrm{CO}$ masers $\mathrm{Ia} / \mathrm{Ib}$ are at $-57.9 /-57.8 \mathrm{~km} \mathrm{~s}^{-1}$. The corresponding $\mathrm{H}_{2} \mathrm{O}$ maser, $\mathrm{M}$, spans the range $[-58.2,-55.2] \mathrm{km} \mathrm{s}^{-1}$, which includes both the $\mathrm{CH}_{3} \mathrm{OH}$ and $\mathrm{H}_{2} \mathrm{CO}$ maser velocities. Hence, the maser counterparts coincide both spatially and kinematically.

\subsection{Comparison with models}

Although the 23.1-GHz methanol masers do coincide with water and formaldehyde masers, they do not coincide with $6.7-\mathrm{GHz}$ or $12.2-\mathrm{GHz}$ methanol masers. Fig. 3 shows that this holds when the positions of the $23.1-\mathrm{GHz} \mathrm{CH}_{3} \mathrm{OH}$ masers are compared to either the masers reported by M00 (right) or by M09 (left). The absolute position uncertainties are 0"'01 or better for M00, and $\sim 0$." 001 for M09. It is not surprising that regions masing at 6.7 or $12.2 \mathrm{GHz}$ might not show 23.1-GHz maser emission - the models of Cragg et al. (2005) indicate that the former transitions mase over a wider range of physical parameters than the latter transition. But it is suprising that 23.1-GHz masers would not be accompanied by 6.7 or $12.2-\mathrm{GHz}$ masers: if conditions are adequate for 23.1-GHz masing, they should also be adequate for 6.7 and 12.2-GHz masing. Moreover, current models of methanol masers (Sobolev et al. 1997a,b; Cragg et al. 2004, 2005) predict brightness temperatures that are orders of magnitude higher for the 6.7 and 12.2- $\mathrm{GHz}$ masers compared to 
the 23.1-GHz maser.

In light of current models, then, the puzzle is why the locations of the 23.1-GHz masers do not also show 6.7 or $12.2-\mathrm{GHz}$ emission. A possible explanation is the non-simultaneity of the observations. However, the observations of M09 were made within days to months of ours, and the maser spots were consistent for all the epochs of M09 (Mark Reid, personal communication).

Changing physical conditions as a function of time are to be expected in the dynamic and turbulent medium of star formation regions (Ballesteros-Paredes et al. 2007, and references therein). In fact, the turbulent medium may be intimately related to the production of the masers (Sobolev et al. 1998). Most of these changes will occur on a dynamic timescale, however, which is typically orders of magnitude longer than astronomical monitoring. Much more rapid change can be produced in the radiation fields. Recent models of H II regions with accretion activity predict the existence of significant variations in the cm free-free flux on timescales as short as a few years (Peters et al. 2010). Fast variations in the radio flux have been reported for IRS1 by Franco-Hernández \& Rodríguez (2004), which is one of the best candidates for an H II region still undergoing accretion (Klaassen et al. 2009; Sandell et al. 2009; Zhu et al. 2010). Moreover, all of the three known 23.1- $\mathrm{GHz} \mathrm{CH}_{3} \mathrm{OH}$ maser regions have background free-free emission detected at radio wavelengths (Menten et al. 1988; Carral et al. 2002; this paper). The extended frequency coverage of the Expanded VLA (EVLA) will facilitate simultaneous multi-transition maser studies of this type of objects at high angular resolution.

Finally, we underscore that if the $N$ 23.1-GHz maser and the 4.8- $\mathrm{GHz} \mathrm{H}_{2} \mathrm{CO}$ are close in space, then similar general conditions may give rise to both rare masers. Boland \& de Jong (1981) developed a pumping model for the IRS $1 \mathrm{H}_{2} \mathrm{CO}$ maser based on free-free emission from the $\mathrm{H}$ II region, and Pratap et al. (1992) concur that this model is adequate to explain the 4.8-GHz maser in IRS 1. Nevertheless, the Boland \& de Jong model also predicts a bright $\mathrm{H}_{2} \mathrm{CO}$ maser at $14.5 \mathrm{GHz}$, and this maser transition was not detected by Hoffman et al. (2003). Furthermore, Arava et al. (2007) showed that this model is not able to explain the $\mathrm{H}_{2} \mathrm{CO}$ maser in another region of highmass star formation: IRAS $18566+0408$. We note that the consideration of non-simultaneous observations applies equally to these coincident masers as it did to the non-coincident methanol masers mentioned above. This caveat notwithstanding, the possible correlation of these two rare maser species may prove helpful in better defining the pumping mechanism of each.

We thank the anonymous referee for comments that significantly improved this paper. We are grateful to the VLA staff for supporting the initial phase of this project via the NRAO program of observing time for university classes. We thank R. Franco-Hernández for providing the 2-cm image; A. Sobolev, J. Ballesteros-Paredes, and M. Reid for helpful discussions; and V. Minier for providing us with maser positions. R. G.-M. acknowledges support from an SMA predoctoral fellowship. E. A. was partially supported by a NRAO Jansky Fellowship. P. H. acknowledges partial support from NSF grant AST-0908901. 


\section{REFERENCES}

Araya, E., Hofner, P., Goss, W. M., Linz, H., Kurtz, S., \& Olmi, L. 2008, ApJS, 178, 330

Araya, E. et al. 2007, ApJ, 669, 1050

Ballesteros-Paredes, J., Klessen, R. S., Mac Low, M.-M., \& Vazquez-Semadeni, E. 2007, Protostars and Planets V, ed. B. Reipurth, D. Jewitt, \& K. Keil (Tucson, AZ: Univ. Arizona Press), 63

Boland, W., \& de Jong, T. 1981, A\&A, 98, 149

Campbell, B. 1984, ApJ, 282, L27

Carral, P., Kurtz, S., Rodríguez, L. F., Menten, K., Cantó, J., \& Arceo, R. 2002, AJ, 123, 2574

Cragg, D. M., Sobolev, A. M., Caswell, J. L., Ellingsen, S. P., \& Godfrey, P. D. 2004, MNRAS, 351,1327

Cragg, D. M., Sobolev, \& Godfrey, P. D. 2005, MNRAS, 360, 533

De Buizer, J. M., \& Minier, V. 2005, ApJ, 628, L151

Franco-Hernández, R., \& Rodríguez, L. F. 2004, ApJ, 604, L105

Galván-Madrid, R., Keto, E., Zhang, Q., Kurtz, S., Rodríguez, L. F., \& Ho, P. T. P. 2009, ApJ, 706, 1036

Gaume, R. A., Goss, W. M., Dickel, H. R., Wilson, T. L., \& Johnston, K. J. 1995, ApJ, 438, 776

Gaume, R. A., Johnston, K. J., Nguyen, H. A., Wilson, T. L., Dickel, H. R., Goss, W. M., \& Wright, M. C. H. 1991, ApJ, 376, 608 (G91)

Hoffman, I. M., Goss, W. M., Palmer, P., \& Richards A. M. S. 2003, ApJ, 598, 1061 (H03)

Hofner, P., Kurtz, S., Churchwell, E., Walmsley, C. M., \& Cesaroni, R. 1994, ApJ, 429, L85

Hutawarakorn, B., \& Cohen, R. J. 2003, MNRAS, 345, 175

Johnston, K. J., Stolovy, S. R., Wilson, T. L., Henkel, C., \& Mauersberger, R. 1989, ApJ, 343, L41

Kameya, O., Koh-Ichiro, M., Ryohei, K., \& Ishiguro, M. 1990, ApJ, 355, 562

Keto E., Zhang, Q., \& Kurtz, S. 2008, ApJ, 672, 423

Klaassen, P. D., Wilson, C. D., Keto, E. R., \& Zhang, Q. 2009, ApJ, 703, 1308

Kraus, S., Balega, Y., Elitzur, M., Hofmann, K.-H., Preibisch, Th., Rosen, A., Schertl, D., Weigelt, G., \& Young, E. T. 2006, A\&A, 455, 521

Kurtz, S., Hofner, P., \& Alvarez, C. 2004, ApJS, 155, 149 
Mauersberger, R., Wilson, T. L., \& Henkel, C. 1986, A\&A, 160, L13

Mehrotra, S. C., Dreizler, H., \& Mäder, H. 1985, Z. Naturforschung, 40, 683

Menten, K. M., \& Batrla, W. 1989, ApJ, 341, 839

Menten, K. M., Johnston, K. J., Wadiak, E. J., Walmsley, C. M., \& Wilson, T. L. 1988, ApJ, 331, L41

Menten, K. M., Wilson, T. L., Walmsley, C. M., Mauersberger, R., Henkel, C., \& Johnston, K. J. 1985, ApJ, 293, L83

Minier, V., Booth, R. S., \& Conway, J. E. 2000, A\&A, 362, 1093 (M00)

Minier, V., Conway, J. E., \& Booth, R. S. 2002, A\&A, 383, 614

Moscadelli, L., Reid, M. J., Menten, K. M., Brunthaler, A., Zheng, X. W., \& Xu, Y. 2009, ApJ, 693, 406

Pestalozzi, M. R., Elitzur, M., Conway, J. E., Booth, R. S. 2004 ApJ, 603, L113

Pestalozzi, M. R., Elitzur, M., \& Conway, J. E. 2009, A\&A, 501, 999

Peters, T., Banerjee, R., Klessen, R., Mac Low, M., Galván-Madrid, R., \& Keto E., 2010, ApJ, 711,101

Pratap, P., Snyder, L. E., \& Batrla, W. 1992, ApJ, 387, 241

Sandell, G., Goss, W. M., Wright, M., \& Corder, S. 2009, ApJ, 699, L31

Sarma, A. P., Troland, T. H., Crutcher, R. M., \& Roberts, D. A. 2002, ApJ, 580, 928

Schilke, P., Walmsley, C. M., \& Mauersberger, R. 1991, A\&A, 247, 516

Sewiło, M., Churchwell, E., Kurtz, S., Goss, W. M., \& Hofner, P. 2004, ApJ, 605, 285

Sobolev, A. M., Cragg, D. M., \& Godfrey, P. D. 1997a, MNRAS, 288, L39

Sobolev, A. M., Cragg, D. M., \& Godfrey, P. D. 1997b, A\&A, 324, 211

Sobolev, A. M., Wallin, B. K., \& Watson, W. D. 1998, ApJ, 498, 763

Wilson, T. L., Walmsley, C. M., Snyder, L. E., \& Jewell, P. R. 1984, A\&A, 134, L7 (W84)

Wynn-Williams, C. G., Becklin, E. E., \& Neugebauer, G. 1974, ApJ, 187, 473

Wyrowski, F. \& Walmsley, C. M. 1996, A\&A, 314, 265

Zhu, L. et al. 2010, in preparation. 
Table 1. Spectra Parameters

\begin{tabular}{ccc}
\hline \hline Parameter & $\mathrm{N}$ & $\mathrm{S}$ \\
\hline Peak $^{\text {a }}\left(\mathrm{mJy} \mathrm{beam}^{-1}\right)$ & $331 \pm 4$ & $97 \pm 3$ \\
$T_{\mathrm{B}, \text { peak }^{\mathrm{a}}(\mathrm{K})}$ & $>1.3 \times 10^{5}$ & $\sim 4 \times 10^{4}$ \\
$\mathrm{~V}_{\mathrm{LSR}}{ }^{\mathrm{a}}\left(\mathrm{km} \mathrm{s}^{-1}\right)$ & $-56.09 \pm 0.02$ & $-59.11 \pm 0.08$ \\
$\Delta V_{\mathrm{FWHM}^{\mathrm{a}}}\left(\mathrm{km} \mathrm{s}^{-1}\right)$ & $1.78 \pm 0.04$ & $2.89 \pm 0.18$ \\
$\theta_{\mathrm{S}}{ }^{\mathrm{b}}(\operatorname{arcsec})$ & $<0.09 \times 0.09$ & $0.11 \times 0.07$ \\
\hline
\end{tabular}

${ }^{a}$ From a Gaussian fit to the spectrum of the component. $1 \sigma$ statistical errors are quoted.

${ }^{\mathrm{b}}$ Spot diameters at half power are from a 2-D Gaussian fit at the peak channel of the component, using the task JMFIT in AIPS. For component $\mathrm{N}$ the value of the minor axis is an upper limit; see $\S 3.1$.

Table 2. Water maser positions and velocities

\begin{tabular}{cccccc}
\hline \hline $\begin{array}{c}\text { Maser } \\
\text { Designation }^{\mathrm{a}}\end{array}$ & $\begin{array}{c}\text { R.A. (J2000) } \\
(\mathrm{sec})^{\mathrm{c}}\end{array}$ & $\begin{array}{c}\text { Dec. }(\mathrm{J} 2000) \\
\left({ }^{\prime \prime}\right)^{\mathrm{b}}\end{array}$ & $\begin{array}{c}\text { Peak } S_{\nu} \\
(\mathrm{Jy})\end{array}$ & $\begin{array}{c}\text { Peak } \mathrm{V}_{L S R} \\
\left(\mathrm{~km} \mathrm{~s}^{-1}\right)\end{array}$ & $\begin{array}{c}\text { Velocity Range } \\
\left(\mathrm{km} \mathrm{s}^{-1}\right)\end{array}$ \\
\hline M1 & 45.305 & 2809.38 & 26.8 & -61.6 & -60.8 to -62.3 \\
M2 & 45.306 & 2809.33 & 0.90 & -58.8 & -58.5 to -59.5 \\
M3 & 45.324 & 2810.57 & 31.7 & -57.8 & -57.3 to -58.5 \\
M4 & 45.340 & 2809.34 & 0.97 & -62.3 & -61.5 to -63.1 \\
M5 & 45.350 & 2810.42 & 138. & -60.1 & -59.5 to -61.1 \\
M6 & 45.351 & 2810.39 & 0.43 & -58.5 & -58.0 to -59.0 \\
M7 & 45.362 & 2810.42 & 0.69 & -56.7 & -55.2 to -58.2 \\
M8 & 45.365 & 2810.32 & 41.7 & -60.0 & -59.2 to -60.8 \\
M9 & 45.377 & 2809.59 & 2.15 & -59.3 & -58.8 to -60.5 \\
M10 & 45.383 & 2810.69 & 0.60 & -53.4 & -53.1 to -54.1 \\
E1 & 46.365 & 2813.65 & 1.03 & -55.0 & -53.4 to -56.2 \\
E2 & 46.930 & 2810.17 & 6.91 & -53.4 & -53.1 to -54.2 \\
E3 & 46.956 & 2809.99 & 0.33 & -61.9 & -61.0 to -62.9 \\
S1 & 44.766 & 2651.22 & 357. & -54.9 & -53.1 to -56.2 \\
S2 & 44.949 & 2649.61 & 3.82 & -57.3 & -56.8 to -57.8 \\
S3 & 44.959 & 2649.73 & 4.47 & -60.8 & -60.1 to -62.6 \\
S4 & 44.964 & 2649.50 & 20.7 & -54.1 & -53.7 to -54.4 \\
S5 & 44.967 & 2649.40 & 11.4 & -56.7 & -56.4 to -57.2 \\
S6 & 44.973 & 2649.43 & 1.49 & -55.7 & -54.9 to -56.8 \\
S7 & 44.973 & 2649.27 & 2.99 & -56.2 & -55.9 to -57.3 \\
S8 & 44.979 & 2649.75 & 2.28 & -62.4 & -62.1 to -62.8 \\
\hline
\end{tabular}

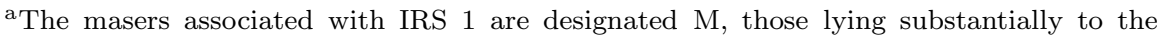
east (about $6^{\prime \prime}$ ) are designated E, and the cluster to the south (corresponding to IRS 11) is designated S. Within each designation the masers are numbered in order of increasing Right Ascension.

${ }^{\mathrm{b}}$ All masers are at $23^{\mathrm{h}} 13^{\mathrm{m}}$ R.A.; only seconds are listed here. Likewise, all masers are at $+61^{\circ}$ Dec.; only minutes and seconds are listed. 
Table 3. Maser Counterparts

\begin{tabular}{|c|c|c|c|c|c|}
\hline $\begin{array}{c}\text { Maser } \\
\text { Designation }\end{array}$ & $\begin{array}{l}\text { R.A. (J2000) } \\
\quad(\text { h m s })\end{array}$ & $\begin{array}{c}\text { Dec. }(\mathrm{J} 2000) \\
(\circ \quad \prime \prime \prime)\end{array}$ & $\begin{array}{l}\text { Abs. Uncertainty } \\
\left({ }^{\prime \prime}\right)\end{array}$ & $\begin{array}{l}\text { Offset }^{\mathrm{a}} \\
\left({ }^{\prime \prime}\right)\end{array}$ & Reference \\
\hline $\mathrm{CH}_{3} \mathrm{OH} 23.1-\mathrm{GHz} N$ & 231345.358 & 612810.45 & 0.05 & - & This paper \\
\hline $\mathrm{H}_{2} \mathrm{CO} 4.8-\mathrm{GHz} I^{\mathrm{b}}$ & 231345.3559 & 612810.444 & 0.003 & 0.02 & Hoffman et al. (2003) \\
\hline $\mathrm{H}_{2} \mathrm{O} M 7$ & 231345.362 & 612810.42 & 0.05 & 0.04 & This paper \\
\hline $\mathrm{CH}_{3} \mathrm{OH} 23.1-\mathrm{GHz} S$ & 231345.365 & 612810.28 & 0.05 & - & This paper \\
\hline $\mathrm{H}_{2} \mathrm{O} M 8$ & 231345.365 & 612810.32 & 0.05 & 0.04 & This paper \\
\hline $\mathrm{H}_{2} \mathrm{O} M 6$ & 231345.351 & 612810.39 & 0.05 & - & This paper \\
\hline $\mathrm{H}_{2} \mathrm{CO} 4.8-\mathrm{GHz} I I^{\mathrm{c}}$ & 231345.351 & 612810.37 & 0.05 & 0.02 & Hoffman et al. (2003) \\
\hline
\end{tabular}

a Offset from the source listed first

${ }^{\mathrm{b}}$ Average position of the $I a$ and $I b$ components reported in Table 4 of H03.

${ }^{\mathrm{c}}$ Position extracted from Figure 5 of H03. 


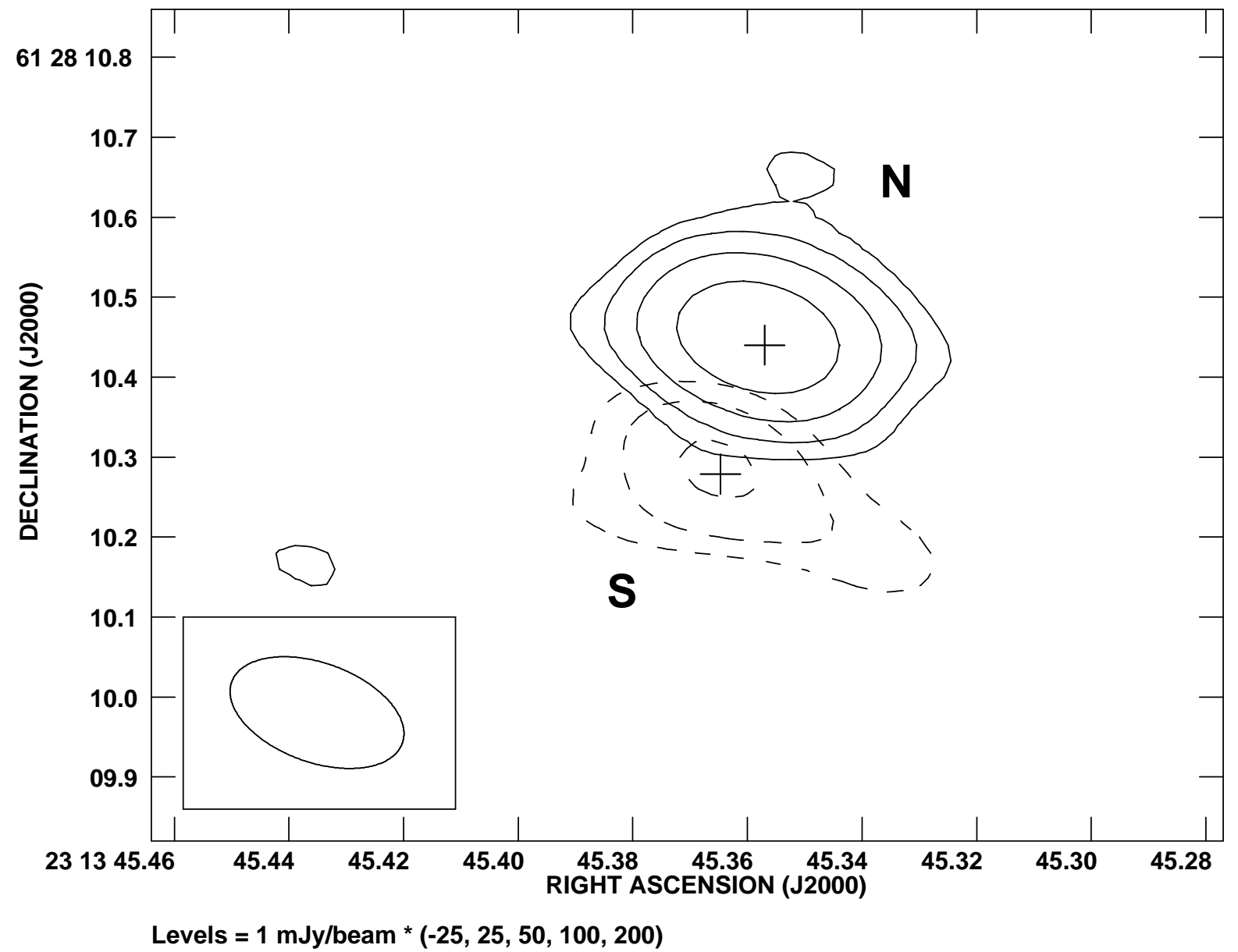

Fig. 1.- Contour plot of the 23.1- $\mathrm{GHz} \mathrm{CH}_{3} \mathrm{OH}$ emission toward NGC 7538 IRS 1. The solid contours show the North component while the dashed contours show the South component. The crosses indicate the peak positions from 2-D Gaussian fits. Both sets of contours, and their respective crosses, were obtained from the peak channel of the corresponding maser. The synthesized beam is shown in the lower left corner. The image rms in each channel is $7 \mathrm{mJy}^{\mathrm{beam}}{ }^{-1}$. 

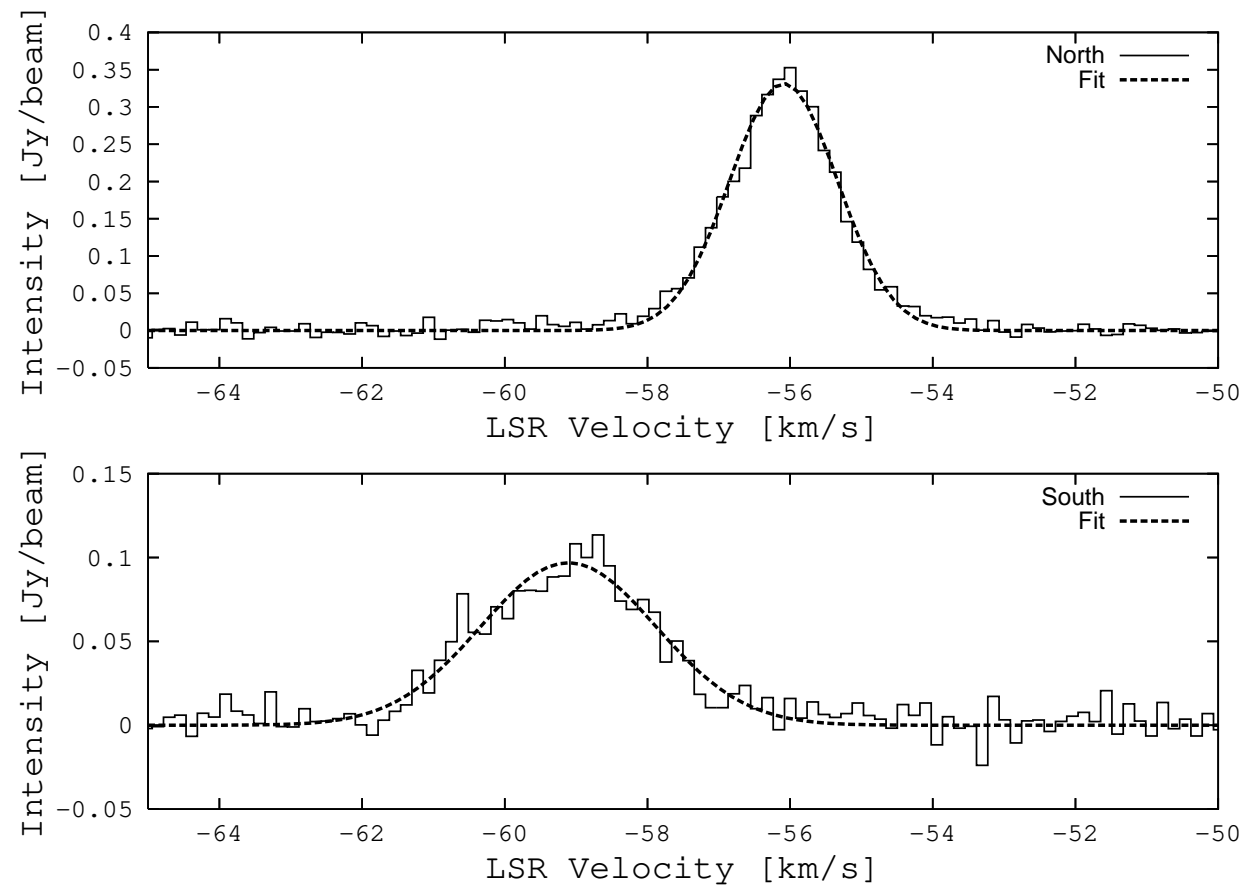

Fig. 2.- $\mathrm{CH}_{3} \mathrm{OH} 9_{2}-10_{1} A^{+}$spectra at $23.1 \mathrm{GHz}$ toward the NGC 7538 IRS 1 region. Top: Spectrum toward the northern maser $N$ (see $§ 3.1$ ). Bottom: Spectrum toward the southern maser $S$. Gaussian fits are plotted with dashed lines. 

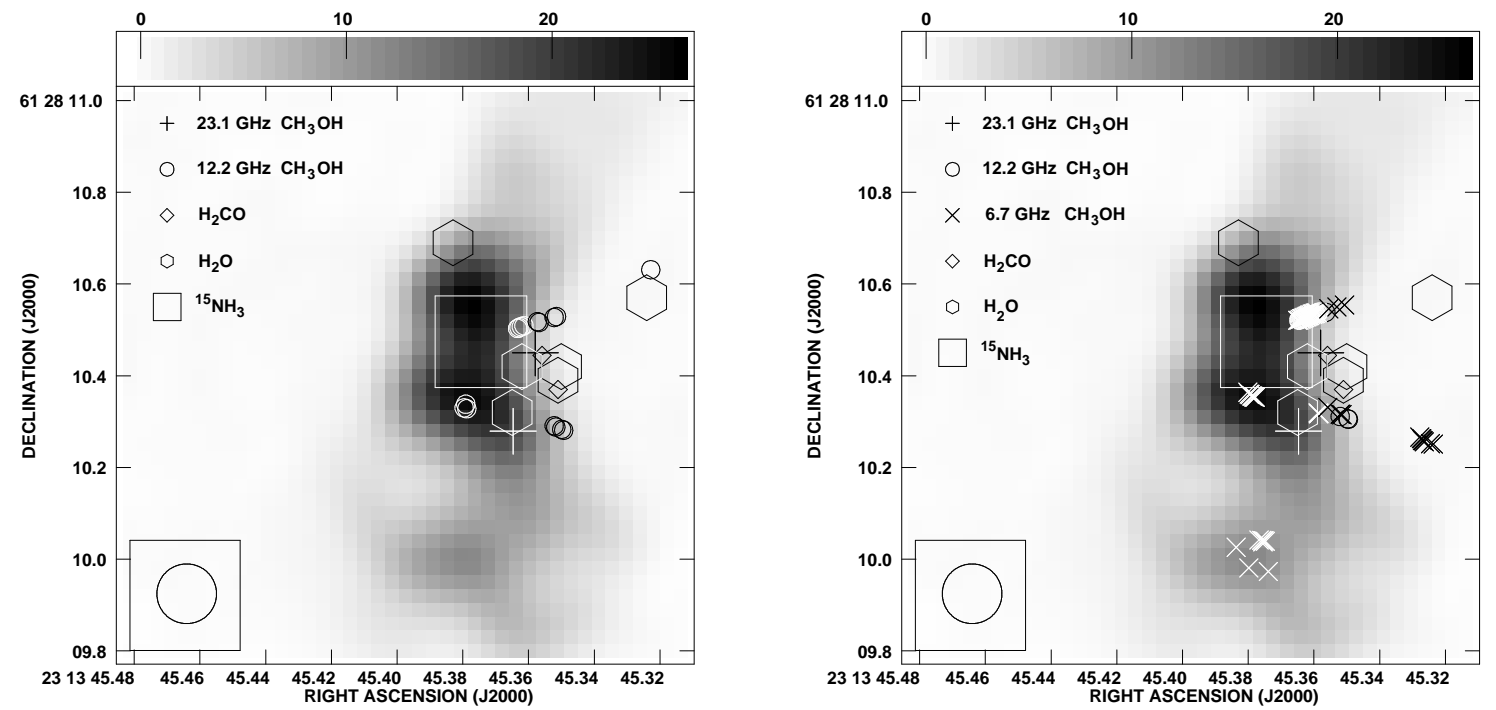

Fig. 3.- Overlay of the 2-cm continuum (gray scale) with masers (symbols) toward NGC 7538 IRS 1. The left panel shows the $12.2-\mathrm{GHz} \mathrm{CH}_{3} \mathrm{OH}$ masers (circles) reported by Moscadelli et al. (2009). The right panel shows the $6.7-\mathrm{GHz}\left(\times\right.$ symbols) and $12.2-\mathrm{GHz}$ (circles) $\mathrm{CH}_{3} \mathrm{OH}$ masers reported by Minier et al. (2000). Both panels are identical in the rest of their content. The square at the core of the nebula denotes the region with multiple ${ }^{15} \mathrm{NH}_{3}$ masers reported by Gaume et al. (1991). The hexagons mark the 22.2- $\mathrm{GHz}_{2} \mathrm{O}$ masers reported in this paper. The diamonds mark the 4.8- $\mathrm{GHz} \mathrm{H}_{2} \mathrm{CO}$ masers observed by Hoffman et al. (2003). The crosses mark the two 23.1- $\mathrm{GHz} \mathrm{CH}_{3} \mathrm{OH}$ masers reported in this paper. The 2-cm continuum image is from Franco-Hernández \& Rodríguez (2004); its synthesized beam is shown in the lower left corner. The symbol size of the new masers reported in this paper corresponds to the upper limit to their absolute position uncertainty $\left( \pm 0.05^{\prime \prime}\right)$. The symbol size for the masers from Hoffman et al. (2003), Minier et al. (2000), and Moscadelli et al. (2009) is larger than their absolute position uncertainty (as small as $\pm 0.001^{\prime \prime}$ for M09). The absolute position uncertainty of the background continuum is similar to that of the new masers we report. The relative uncertainties for a given observation are smaller than the symbols for all cases. 